\title{
Nuclear Structure from Radioactive Decay
}

DE92 003673

Annual Progress Report

U. S. Department of Energy

Grant DE-FG05-87ER40330

\author{
J. L. Wood \\ Associate Professor of Physics and Principal Investigator
}

September 30, 1991

School of Physics

Georgia Institute of Technology

Atlanta, Georgia 30332

Telephone: (404) 894-5262

\section{DISCLAIMER}

\begin{abstract}
Ti,is renort was prepared as an account of work sponsored by an agency of the United States Tisis report was prepared as an accume Government nor any agency thereof, nor any of their Government. Neither the United States Government or assumes any legal liability or responsiemployees. inakes any warranty, express or implied, or assumes any legal liability or responsibility for the accuracy, completeness, or usefulness of any information, apparatus, product, or process disclosed, or represents that its use would not infringe privately owned rights. Reference herein to any specific commercial product, process, or service by trade name, trademark, manufacturer, or otherwise does not necessarily constitute or imply its endorsement, recommendation, or favoring by the United Staies Government or any agency thereof. The views and opinions of authors expressed herein do not necessarily state or reflect those of the United States Government or any agency thereof.
\end{abstract}


Table of Contents

page

1.0 Introductory overview. . . . . . . . . . . . . . . 1

2.0 Experimental Program in the $z=82$ Region............ 2

2.1 Neutron-Deficient Iridium Isotopss. . . . . . . . . . 3

2.2 Neutron-Deficient Platinum Isotopes . . . . . . . . . 3

2.3 Neutron-Deficient Gold Isotopes ............. . 3

2.4 Neutron-Deficient Mercury Isotopes. . . . . . . . . . 4

2.5 Neutron-Deficient Thallium Isotopes ........... 4

2.6 Neutron-Deficient Lead Isotopes . . . . . . . . . . 5

3.0 Experimental Program in the $2>58, \mathrm{~N}<73$ Region. . . . . . . . 6

3.1 Neutron-Deficient Samarium Isotopes . . . . . . . . . 6

3.2 Neutron-Deficient Promethium Isotopes . . . . . . . 6

3.3 Neutron-Deficient Neodymium Isotopes. . . . . . . . . 7

3.4 Neutron-Deficient Praseodymium Isotopes . . . . . . . . 7

4.0 Nuclear Systematics and Models . . . . . . . . . . . . 7

5.0 Overseas Trips ...................... 9

6.0 Personnel. . . . . . . . . . . . . . . . . . 10

7.0 Summary of Publications, Preprints, Abstracts, and Presentations at Conferences. . . . . . . . . . . . . . . 11 


\subsection{Introductory Overview}

The most exciting development this year was the successful routine operation at UNISOR of a new ion source design that provides high yields of short-lived isotopes of $\mathrm{Pr}, \mathrm{Nd}, \mathrm{Pm}, \mathrm{Sm}$, and $\mathrm{Eu}$. This provides unprecendented access to the nuclear structure of the $2 \gtrsim 58, N \lesssim 78$ region.

A major review of shape coexistence in even-mass nuclei has been completed and submitted to Physics Reports.

Part of our experimental program continues to focus on shape coexistence and electric monopole (EO) transitions in nuclei. Details of coexisting bands and associated electric monopole transitions in ${ }^{190} \mathrm{Hg}$ and ${ }^{195} \mathrm{~Pb}$ have been published in Physical Review $C$ and Nuclear Physics A, respectively.

A very high statistics study of the ${ }^{194} \mathrm{Bi} \rightarrow{ }^{194} \mathrm{~Pb}$ decay scheme was made in search of low-spin members of the superdeformed band in ${ }^{194} \mathrm{~Pb}$.

Details of the discovery of pure electric monopole transitions in ${ }^{185} \mathrm{Pt}$ -- an unprecented nuclear decay process for an odd-mass nucleus -- has been submitted for publication in Physical Review Letters.

Theoretical investigations have continued in collaboration with Prof. $K$. Heyde, Institute of Nuclear Physics, Gent, Belgium and with Prof. D. J. Rowe, University of Toronto. Papers have been published on intruder states and shape coexistence near $N=20$ and on the $S U(3)$ structure of rotational states in heavy det'ormed nuclei in Journal of Physics G: Nuclear Physics and in Nuclear Physics A, respectively.

This year the contract has supported, besides the principal investigator (two months, full time), a research scientist (twelve months, one-twelfth time), and three graduate students (two for twelve months, hasf time; one for six months, half time). A fourth student is pursuing a Ph.D. degree on topics described in this proposal with full financial support from the Georgia Tech School of Physics. 


\subsection{Experimental Program in the $Z=82$ Region}

A major area of activity is the study of neutron-deficient nuclei around the $2=82$ shell closure, with special emphasis on the levels of the odd-mass Ir, $\mathrm{Pt}, \mathrm{Au}, \mathrm{Hg}, \mathrm{Tl}$, and $\mathrm{Pb}$ isotopes. A crucial ingredient of this work is the systematic investigation of low-energy structural features of sequences of isotopes (and isotones) over many adjacent mass numbers. This has a two-fold purpose. First, it enables us to build up a very complete picture of the nuclear structure that connects the regions of stable nuclei (where, e.g., transfer reactions and Coulomb excitation permit the measurement of detailed spectroscopic properties) with regions far from stability, where detailed spectroscopic information is very limited. Second, it provides a map of the excitation degrees of freedom as a function of the changing proton and neutron number over broad mass regions. The mass region under study possesses the richest variety of nuclear excitations observed anywhere on the mass surface. It includes the $Z=82$ shell closure, and is bounded by the strongly deformed nuclei with $Z \leq 76$ and the $N=126$ shell closure. Between $Z=76$ and $Z=8$ ? there is a shape transition from strongly deformed prolate axial symmetry through oblate axial asymmetry to spherical symmetry. This transition has alternatively been described in terms of the interacting boson approximation (IBA) as a transition from the $S U(3)$ through the $O(6)$ limiting algebraic symmetries of the IBA Hamiltonian. Finally, and most dramatically of all, an island of ground-state deformation has been established adjacent to the $2=82$ closed shell for the very neutron-deficient isotopes of $\mathrm{Hg}$, $\mathrm{Au}$, and $\mathrm{Pt}$. This deformation is now understood in terms of proton intruder orbitals which give rise to the large deformation $(\beta \sim 0.27)$ through an increased proton valence space and the proton-neutron residual interaction. The experiments described lave all (except part of one) been done at UNISOR. 


\subsection{Neutron-Deficient Iridium Isotopes}

The $\gamma-\gamma$ and $e-\gamma$ coincidence data for the decays $181,183,185 \mathrm{Pt} \rightarrow$ Ir are in the process of being analyzed. The iridium isotopes border the region of shape coexistence defined by $78 \leq 2 \leq 83$. So far, shape coexistence has not been found in the iridium isotopes. It is being looked for in this work through the EO transition "fingerprint". Limited operations at Holifield have precluded a study of ${ }^{179} \mathrm{Pt} \rightarrow \mathrm{Ir}$. This work constitutes part of the Ph.D. thesis work of Ms. K. Jentoft-Nilsen (Ga. Tech., School of Physics).

\subsection{Neutron-Deficient Platinum Isotopes}

The $r-\gamma$ and $e-r$ coincidence data for the decays $181,183,185 \mathrm{Au} \rightarrow$ Pt are in the process of being analyzed. As noted in the introduction, pure electric monopole transitions have been discovered in ${ }^{185} \mathrm{Pt}$. This is an unprecented nuclear decay process for an odd-mass nucleus where, because spins must be $\geq 1 / 2$, M1 multipolarity is always possible. Details have been submitted for publication in Physical Review Letters. This work constitutes part of the Ph.D. thesis work of Mr. J. Schwarzenberg (Ga. Tech., School of Chemistry) and is in collaboration with Prof. E. F. Zganjar (Louisiana State University)

The $\gamma-\gamma$ and $e-\gamma$ coincidence data for the decay ${ }^{184} \mathrm{Au} \rightarrow$ Pt are also in the process of being analyzed. Nuclear orientation data for this decay are also being analyzed. We observed two $K^{\pi}=0^{+}$and two $K^{\pi}=2^{+}$bands at low energy with interband EO transitions. We interpret this in terms of coexisting triaxial shapes. A letter-type publication is in preparation. This work is in collaboration with Prof. K. S. Krane (Oregon State Univ.)

A study of the a decay of ${ }^{185} \mathrm{Pt}$ has been published in Physical Review $\mathrm{C}$.

\subsection{Neutron-Deficient Gold Isotopes}

The $\gamma-\gamma$ and e-r coincidence data for the decays $187,{ }^{191} \mathrm{Hg} \rightarrow$ Au are in the process of being analyzed. The study of the $189 m, \mathrm{~B}_{\mathrm{HB}_{\mathrm{B}}} \rightarrow \mathrm{Au}$ decay schemes is 
being prepared for publication. These studies have throe goals: mapping the shape coexisting structures in the neutron-deficient Au isotopes; establishing detailed systematics for these isotopes; and testing particle-core coupling models for these nuclei. This work is in collaboration with E. F. Zganjar. A study of the a decay of ${ }^{185}$ Au has been published in Physical Review C.

\subsection{Neutron-Deficient Mercury Isotopes}

Our discovery of coexisting bands and EO transitions in ${ }^{190} \mathrm{Hg}$ has been published in Physical Review $C$.

The study of the ${ }^{189 \mathrm{~m}_{\mathrm{T}}} \mathrm{T} \rightarrow \mathrm{Hg}$ decay scheme is being prepared for publication. Coincidence data obtained for the $191 \mathrm{~m}_{\mathrm{T} \ell} \rightarrow \mathrm{Hg}$ decay scheme are being analyzed.

Further analysis of the ${ }^{190} \mathrm{~T} \ell \rightarrow \mathrm{Hg}$ coincidence data and ${ }^{192} \mathrm{~T} \ell \rightarrow \mathrm{Hg}$ coincidence data is in progress. The goal of these studies is the characterization of shape coexistence in the $\mathrm{Hg}$ isotnpes.

This work is in collaboration with E. F. Zganjar.

\subsection{Neutron-Deficient Thallium Isotopes}

A study of the ${ }^{195 m, 8_{\mathrm{Pb}}} \rightarrow$ Te decay schemes has been prepared for publication (this was documented in the last year's Annual Report). Other studies (see last year's Annual Report) in the neutron-deficient thallium isotopes have been inactive in the past year. This work is in collaboration with Prof. C. R. Bingham, University of Tennessee. 


\subsection{Neutron-Deficient Lead Isotopes}

Our study of the ${ }^{195} \mathrm{Bi} \rightarrow \mathrm{Pb}$ decay scheme has been published in Nuclear Physics A. The work reports the discovery of coexisting shapes and EO transitions.

Very high statistics $\gamma-\gamma$ and $e-\gamma$ coincidence data were obtained in a study of the ${ }^{194} \mathrm{Bi} \rightarrow \mathrm{Pb}$ decay scheme. These data are in the process of being analyzed. The goals of this study are to obtain detailed information on coexisting shapes in ${ }^{194} \mathrm{~Pb}$ and to ascertain if the low-spin members of the superdeformed band, observed by in-beam r-ray spectroscopy, can be populated in radioactive decay. This work constitutes part of the Ph. D. thesis work of Mr. M. Jarrio (Ga. Tech., School of Physics), and is a collaboration with Dr. E. A. Henry (Lawrence Livermore Liboratory). 


\subsection{Experimental Program in the $2>58, N<78$ Region}

A systematic study of low-spin states in nuclei in and bordering the deformed region with $Z \gtrsim 58, N \lesssim 78$ has been started. This is a new region of deformation and almost nothing is known about low-spin states in this region. Extensive in-beam studies have been made in this region, but such studies see mainly high-spin states. This has been a difficult region for decay scheme studies in the past because the elements involved are relatively refractory and, thus, not quickly released from an ion source, hence precluding on-line isotope separation. This has now changed with the commissioning of a highly-efficient thermal ion source.

The new ion source has been constructed following a design provided by Dr. R. Kirchner of GSI, Darmstadt, Germany. It can operate at temperatures of up to $2400^{\circ} \mathrm{C}$. In routine operation it can deliver high yields of rare-earth elements even when the half life is only a few seconds. For example, it has delivered the highest-ever statistical quality coincidence data for an odd-mass nucleus decay study at UNISOR, in our investigation of the ${ }^{137} \mathrm{Nd} \rightarrow \mathrm{Pr}$ decay. The ion source development constitutes part of the Ph.D. thesis work of Mr. J. Breitenbach (Ga. Tech, School of Physics).

\subsection{Neutron-Deficient Samarium I sotopes}

We have obtained $\gamma-\gamma$ and $e-\gamma$ coincidence data for the decay ${ }^{137}$ Eu $\rightarrow$ ${ }^{137} \mathrm{Sm}$. These data greatly improve on our earlier study of this decay using a lower temperature ion source with the injection of carbon tetrachloride. The data are in the process of being analyzed. In the first phase of this program, we are studying the levels of ${ }^{137} \mathrm{Sm}$ to establish systematic trends of low-energy low-spin states of odd-neutron nuclei in this region.

\subsection{Neutron-Deficient Promethium Isotopes}

We have obtained $\gamma-\gamma$ and $e-\gamma$ coincidence data for the ${ }^{135,137} \mathrm{Sm} \rightarrow \mathrm{Pm}$ 
decays. The ${ }^{137} \mathrm{Sm} \rightarrow \mathrm{Pm}$ data are a great improvement on our earlier study using a lower-temperature ion source with the injection of carbon tetrachloride. The data are in the process of being analyzed. This work constitutes part of the Ph.D. thesis work of J. Breitenbach.

\subsection{Neutron-Deficient Neodymium Isotopes}

We have obtained $r-\gamma$ and $e-r$ coincidence data for the $133,135,137 \mathrm{Pm} \rightarrow \mathrm{Nd}$ decays. The data are in the process of being analyzed. We disagree with the assigned half life of ${ }^{133} \mathrm{Pm}$ in the Chart of the Nuclides and, from mass separation and $x$-ray coincidences, thus, claim its discovery as a $\sim 5 s$ activity with $\gamma$ rays of 118,180 , and $290 \mathrm{keV}$. This work constitutes part of the Ph.D. thesis work of J. Breitenbach.

\subsection{Neutron-Deficient Praseodymium Isotopes}

We have obtained $\gamma-\gamma$ and e-r coincidence data for the $133,135,137 \mathrm{Nd} \rightarrow \mathrm{Pr}$ decays. The data are in the process of being analyzed. This work constitutes part of the Ph.D. thesis work of J. Breitenbach.

\subsection{Nuclear Systematics and Models}

A study of intruder states and shape coexistence in the region $N \sim 20,2$ $\sim 12$ has been published in Journal of Physics G: Nuclea Physics.

A study of the SU(3) structure of rotational states in heavy deformed nuclei has been published in Nuclear Physics A.

A major review of shape coexistence in doubly-even nuclei, at the invitation of Physics Reports, has been completed and submitted. This was done in collaboration with Prof. W. Nazarewicz (Univ. of Warsaw, Poland, and Joint Institute for Heavy Ion Research, Oak Ridge), Prof. K. Heyde (Institute of Nuclear Physics, Gent, Belgium), and Dr. M. Huyse and Dr. P. Van Duppen 
(Instituut voor Kern- en Stralingsfysika, Katholieke Universiteit, Leuven, Belgium).

A paper on the systematics of EO transitions in relationship to shape coexistence has been published in Nuclear Physics A.

The theoretical study of the SU(3) structure of rotational states in heavy deformed nuclei is continuing with an extension to odd-mass nuclei. One of the goals of this program is to explore the microscopic structure of rotational bands associated with shape coexistence. This program is in collaboration with Prof. D. J. Rowe (Univ. of Toronto) and constitutes part of the Ph.D. thesis work of M. Jarrio.

An extensive program of data compilation, evaluation, and systema:ization for the $Z \gtrsim 58, N \lesssim 78$ region has been initiated in support of the experimental program described in Section 3. Calculations in the framework of the Lund model have been initiated for that region to complement these programs. This is being pursued in collaboration with Prof. P. Semmes (Tennessee Technological Institute, Cookeville) and constitutes part of the $P ! 1 . D$. thesis work of $\mathrm{J}$. Breitenbach. 


\subsection{Overseas $\operatorname{Trips}$}

No overseas trips were made at DOE expense or on DOE supported time this year. 


\subsection{Personnel}

\section{Senior Staff}

Dr. J. L. Wood, Associate Professor, Principal Investigator, Full time, 2 months.

Dr. R. A. Braga, Research Scientist, One-twelfth time, 12 months.

\section{Graduate Students}

Mr. Johannes Schwarzenberg, Ph.D. thesis work. ${ }^{+}$Half-time, 6 months.

Mr. Martin Jarrio, Ph.D. thesis work. Half-time, 12 months.

Mr. Jurgen Breitenbach, Ph.D. thesis work. Half-time, 12 months.

Ms. Kristi Jentoft-Nilsen, Ph.D. thesis work. Not supported by DOE.

${ }^{+}$Award of Ph.D. expected in this contract year. 
7.0 Summary of Publications, Preprints, Abstracts, and Presentations at Conferences, and Symposia 1991

1. "The SU(3) Structure of Rotational States in Heavy Deformed Nuclei", M. Jarrio, J. L. Wood, and D. J. Rowe, Nucl. Phys. A528, 409 (1991).

2. "Shape Coexistence in ${ }^{190} \mathrm{Hg}$, M. O. Kortelahti, E. F. Zganjar, J. L. Wood, C. R. Bingham, H. K. Carter, K. S. Toth, J. H. Hamilton, J. Kormicki, L. Chaturvedi, and W. B. Newbolt, Phys. Rev. C43, 484 (1991).

3. "Intruder State: and Shape Coexistence in the Region $N \sim 20,2 \sim 12 ", K$, Heyde and J. L. Wood, J. Phys. G: Nucl. Part. Phys. 17, 135 (1991).

4. "Decay of Mass-Separated $3.0 \mathrm{~min}{ }^{195 \mathrm{~B}_{\mathrm{Bi}}}$ to Levels in ${ }^{195} \mathrm{~Pb}$ and Shape Coexistence in the Neutron-Deficient Odd-Mass Pb Isotopes", J. C. Griffin, R. A. Braga, R. W. Fink, J. L. Wood, H. K. Carter, R. L. Mlekodaj, C. R. Bingham, E. Coenen, M. Huyse, and P. Van Duppen, Nucl. Phys. A530, 401 ( 1991$)$.

5. "Identification of ${ }^{185}$ Pt a Activity and Study of ${ }^{185}$ Au a Decay", C. R. Bingham, M. B. Kassim, M. 2hang, Y. A. Akovali, K. S. Toth, W. D. Hamilton, H. K. Carter, J. Kormicki, J. von Schwarzenberg, and M. M. Jarrio, Phys. Rev. C44, 1208 (1991).

6. "Electric Monopole Transitions and Shape Coexistence in Nuclei", E. F. Zganjar and J. L. Wood, Nucl. Phys. A520, 427c (1990).

7. "Spectroscopic Techniques in the Study of Radioactive Nuclei Far From Stability: Results for the $Z=82$ Region", E. F. Zganjar, J. L. Wood, and J. Schwarzenberg, in Exotic Nuclear Spectroscopy, ed. W. C. McHarris (Plenum Press, New York, 1990), p. 39.

8. "Coexistence in Even-Mass Nuclei", J. L. Wood, K. Heyde, W. Nazarewicz, M. Huyse, and P. Van Duppen, preprint (review invited by Physics Reports: submitted). 
9. "Pure Electric Monopole Transitions in an Odd-Mass Nucleus", J. Schwarzenberg, J. L. Wood, and E. F. Zganjar, preprint (submitted to Phys. Rev. Lett.).

10. "Shape Coexistence in Nuclei", [abstract] Symposium on Recent Advances in Nuclear Structure Research, American Chemical Society Meeting, Atlanta, April 14-19 (1991).

11. "Detailed Nuclear Structure Studies Fàr From Stability", [abstract] Second International Conference on On-Line Nuclear Orientation and Related Topics, Oak Ridge, October 16-19 (1991).

12. "Shape Coexistence in Nuclei", [invited talk] Symposium on Recent Advances in Nuclear Structure Research, American Chemical Society Meeting, Atlanta, April 1/4-19 (1991).

13. "Shape Coexistence Along the $N=2$ Line", [invited talk] International Symposium on Group Theory and Special Symmetries in Nuclear Physics in honor of K. T. Hecht, Ann Arbor, Michigan, Sept. 19-21 (1991). 14. "Spectroscopy Results at UNISOR in the Eighties", [invited talk] Symposium on Reflections and Directions in Low Energy Heavy Ion Physics, Oak Ridge, October 14-15 (1991).

15. "Detailed Nuclear Structure Studies Far From Stability", [invited talk] Second International Conference on On-Line Nuclear Orientation and Related Topics, Oak Ridge, October 16-19 (1991). 

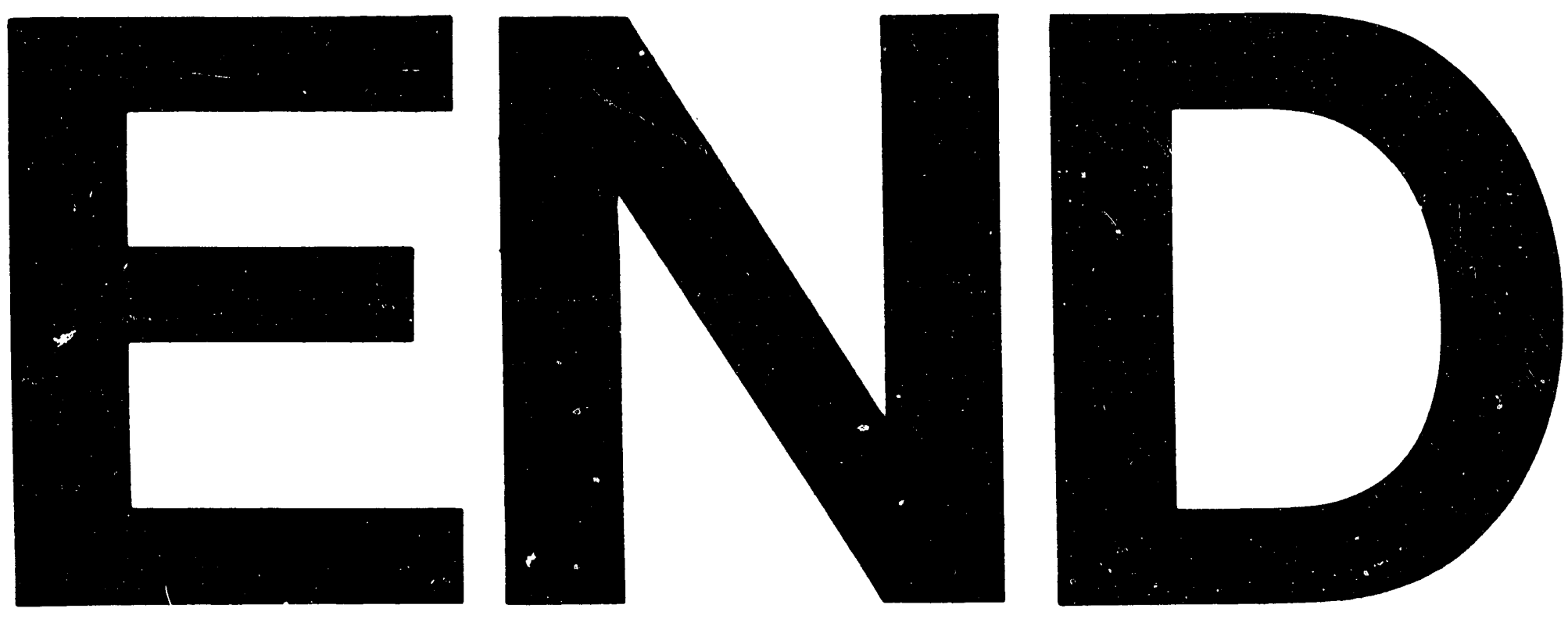

†
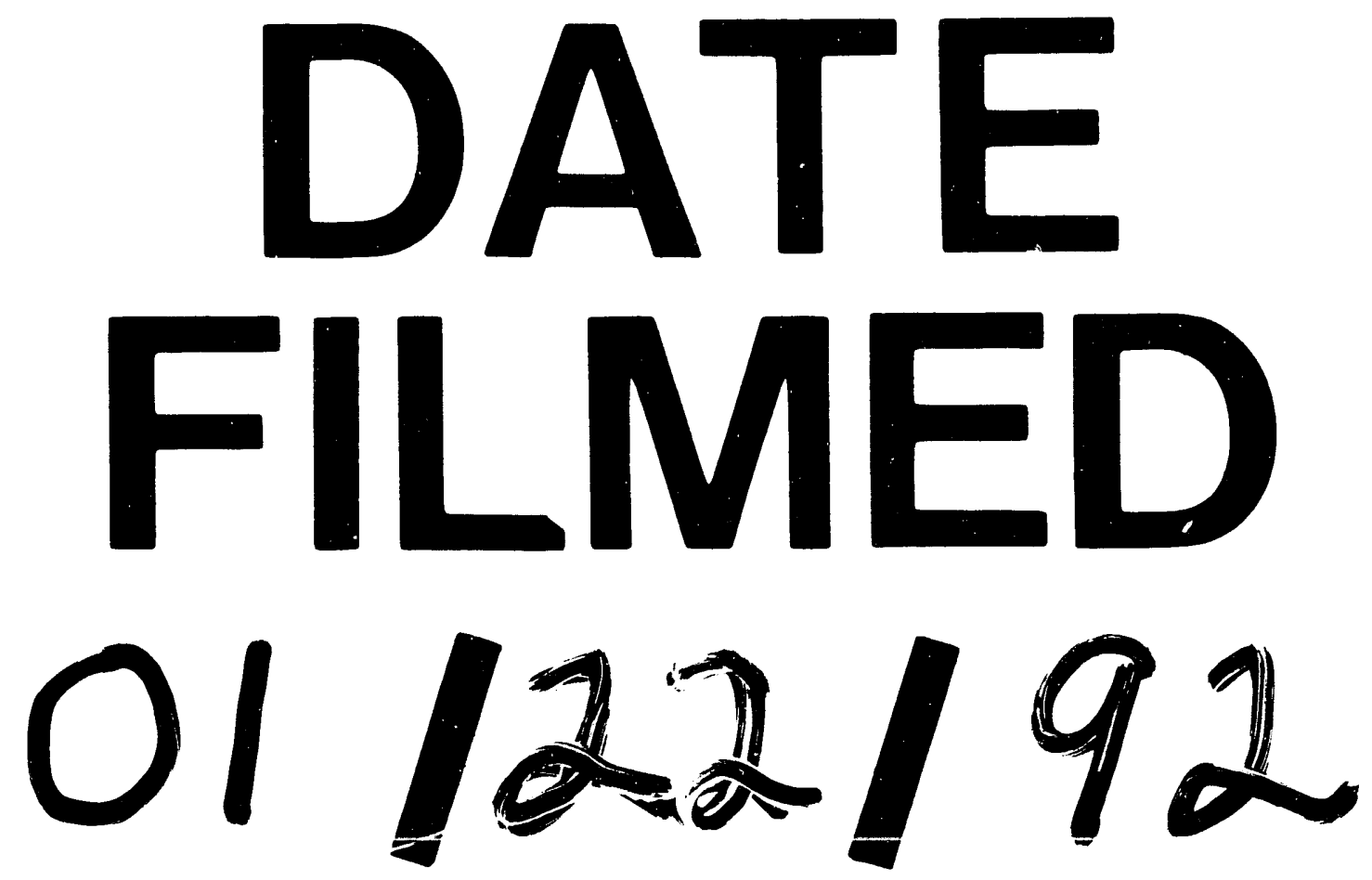
